P81 FEASIBILITY OF MEASURING LUNG CLEARANCE INDEX (LCI) IN A CLINIC SETTING IN PRESCHOOL CHILDREN WITH A RANGE OF AIRWAY DISEASES

${ }^{1} B$ Downing, ${ }^{2} S$ Irving, ${ }^{1} Y$ Bingham, ${ }^{1} \mathrm{~L}$ Fleming, ${ }^{1} \mathrm{~A}$ Bush, ${ }^{1} \mathrm{~S}$ Saglani. ${ }^{1}$ Imperial College London, London, UK; ${ }^{2}$ Royal Brompton and Harefield Trust, London, UK

\subsection{6/thoraxjnl-2015-207770.218}

Introduction and objectives LCI is a measurement of lung function (in particular distal airway disease) derived from the multiple breath washout (MBW) test (Eur Resp J 2013; 41:507-22). Although practical in a research setting, feasibility in a clinic setting (with limited time and without using sedation) in young children is not known. We looked at success rates of LCI, and LCI0.5 (a shortened washout which can be accomplished more quickly) in preschool children (aged 2-6 years) with recurrent wheeze (Eur Resp J 2008; 32:1096-110), cystic fibrosis (CF), recurrent cough/infections, and healthy controls. Our hypothesis (based on other research performed in this field (Thorax 2012; 68:586-587) was that shortened LCI0.5 would be more feasible than full LCI, and that the test would be more feasible in older preschool children than younger.

Methods 62 preschool children median age 3.9 (2.07-5.95) years, 34 male, $(\mathrm{n}=21$ with wheeze, $\mathrm{n}=11 \mathrm{CF}, \mathrm{n}=2 \mathrm{PCD}, \mathrm{n}$ $=22$ other, $\mathrm{n}=5$ healthy controls) performed MBW test during a routine outpatient visit. Wheeze was doctor diagnosed or parent reported via wheeze questionnaire.

Results $66 \%$ of children successfully completed either the LCI or LCI0.5. Completion according to age group is shown in Figure 1. LCI success rate in wheezers was 67\%, healthy controls $100 \%$, CF $82 \%$, PCD 100\% and recurrent cough/infections 50\%. Success rate was identical between males and females (61\%), and was similar comparing LCI0.5 (42/62) to full LCI (38/62). Three of the four that only completed LCI0.5 were less than 3.5 years old. Conclusions LCI is a feasible test in the clinic setting for preschool children; however success rates under 3 years of age in all disease groups are very low. Use of the shortened washout (LCI0.5) marginally improves success rates, but may improve test completion in the youngest children.

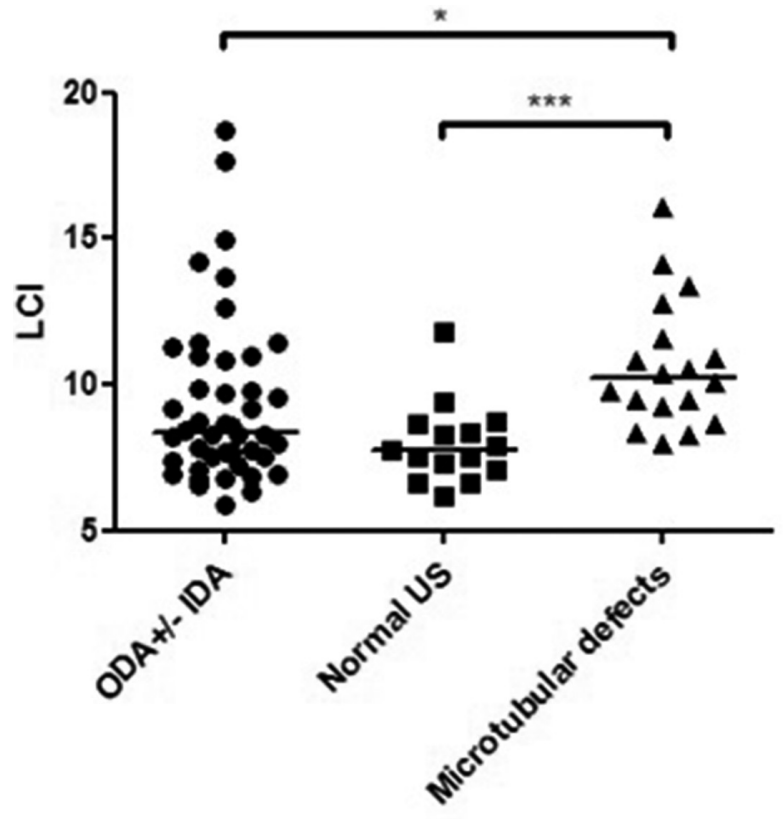

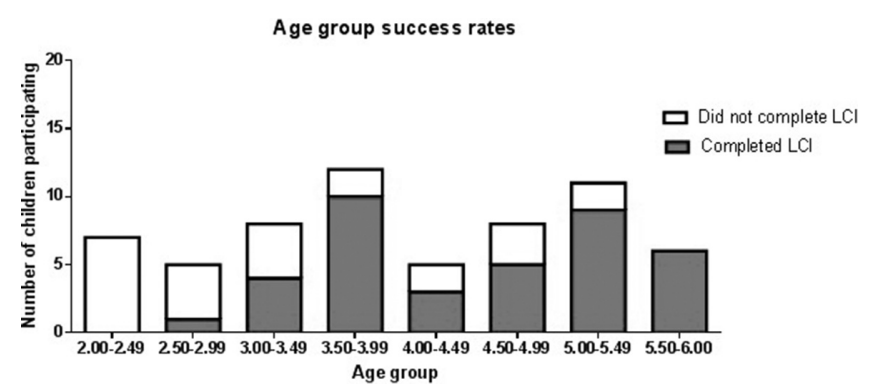

Abstract P81 Figure 1

\section{P82 LUNG CLEARANCE INDEX (LCI) AND GENOTYPE- PHENOTYPE CORRELATIONS IN PRIMARY CILIARY DYSKINESIA (PCD)}

${ }^{1} \mathrm{~S}$ Irving, ${ }^{1} \mathrm{M}$ Dixon, ${ }^{1} \mathrm{~S}$ Ollosson, ${ }^{1} \mathrm{C}$ Hogg, ${ }^{1} \mathrm{~A}$ Shoemark, ${ }^{2} \mathrm{~A}$ Bush. ${ }^{1}$ Royal Brompton and Harefield NHS Foundation, London, UK; ${ }^{2}$ Imperial College, London, UK

\subsection{6/thoraxjnl-2015-207770.219}

Introduction and objectives Mutation type may affect clinical phenotype in PCD, as shown by differences in forced expiratory volume in $1 \mathrm{~s}\left(\mathrm{FEV}_{1}\right)$ (AJRCCM 2015;191:316-324). LCI, measured using multi-breath washout (MBW) is raised in PCD (AJRCCM 2013;188:545-549) but the relative sensitivities of the two physiological measurements is disputed (Thorax 2015;70: 339-345, and 305-306). We hypothesised that LCI would be more sensitive to genotype-phenotype differences in PCD.

Methods MBW (using sulphur hexafluoride MBW with a photoacoustic gas analyser) and spirometry were performed in 77 PCD patients (mean age 16.4 years (range 4-62.2), 33 males, mean $\mathrm{FEV}_{1} \mathrm{z}$ score -2.09 (range -5.33-1.59)). 44 had outer dynein arms (ODA) defects, or both inner (IDA) and ODA, 18 had microtubular defects (either transposition or microtubule disorganisation with absent IDA), 15 had normal ultrastructure (diagnosis made on either genetics $(n=10)$, low nasal NO, clinical phenotype and consistent dyskinesia on light microscopy

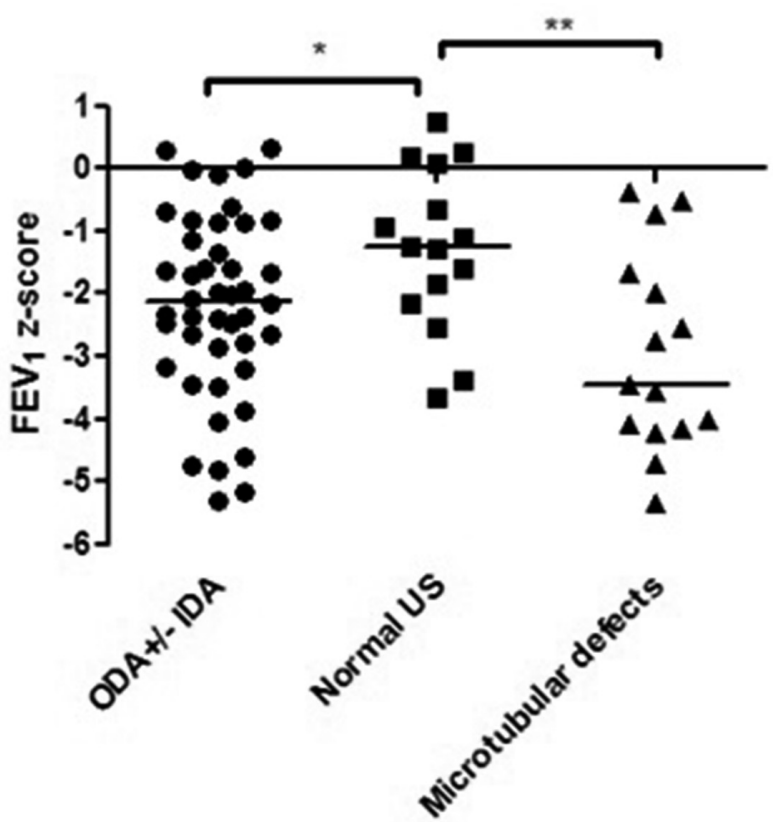

Abstract P82 Figure $1 \mathrm{LCl}$ is worst in other defects group than dynein arm defects $(p=0.01)$ or normal ultrastructure $(p=0.0002)$. FEV $\mathrm{V}_{1}$ is better in normal ultrastructure than dynein arm $(p=0.04)$ and other defects $(p=0.007)$ 
$(\mathrm{n}=1)$, or low nasal NO, clinical phenotype and an affected sibling $(\mathrm{n}=3))$. There was no significant difference in age or gender composition between the 3 groups.

Results Patients with normal ultrastructure had significantly higher $\mathrm{FEV}_{1}$ and lower LCI, indicating milder disease. Those with ODA +/- IDA had a more normal LCI than those with microtubular defects (Figure 1), but similar $\mathrm{FEV}_{1}$.

Conclusions PCD patients with normal ultrastructure have the milder disease, and those with microtubular defects more severe. Differences were more apparent on LCI than $\mathrm{FEV}_{1}$, suggesting LCI may be more sensitive to worse distal small airway disease in PCD.

\section{P83 REMOTE PULMONARY FUNCTION TESTING - COMPUTER GAMING IN THE RESPIRATORY WORLD}

${ }^{1} \mathrm{C}$ Sharp, ${ }^{2} V$ Soleimani, ${ }^{2} \mathrm{~S}$ Hannuna, ${ }^{2} \mathrm{M}$ Camplani, ${ }^{2} \mathrm{D}$ Damen, ${ }^{3} \mathrm{~J}$ Viner, ${ }^{2} \mathrm{M}$ Mirmehdi, 1J Dodd. 'Academic Respiratory Unit, University of Bristol, Bristol, UK; ${ }^{2}$ Visual Information Laboratory, Faculty of Engineering, University of Bristol, Bristol, UK; ${ }^{3}$ Respiratory Physiology, North Bristol NHS Trust, Bristol, UK

\subsection{6/thoraxjnl-2015-207770.220}

Introduction Lung function testing by spirometry has remained unchanged for over 50 years, despite limitations including patient technique, discomfort, cost and training. Non-invasive, remote lung volume measurement is an alternative approach. This has been explored in the past using structured light, accelerometers and impedance pneumography, but these have often been costly and of limited validity.

We use a novel approach to remote assessment ( $\sim 2$ metres) using a 3D time-of-flight depth camera - similar to those found in many home gaming consoles. This pilot developmental data was generated from patients in a clinical setting.

Methods Patients were recruited from a general respiratory physiology laboratory. Spirometry was performed according to ATS/ ERS standards using an unmodified pneumotachograph (nSpire Health, Longmont, CO, USA). A Kinect V2 time-of-flight depth sensor (Microsoft, Redmond, WA, USA) was used to reconstruct 3D models of the subject's thorax to estimate volume-time and flow-time curves for both Forced and Slow Vital Capacity and their associated measurements (Figure 1, technical details in ${ }^{1}$ ).

These results were correlated with simultaneous recordings from the pneumotachograph, and error values calculated to assess the accuracy of the technique.

Results Data were available from 53 patients, with 40 having usable data. Mean age 62.8 yrs (SD 16.2), BMI of 26.8 (SD 5.5). $41.5 \%$ male. $54.7 \%$ of patients had obstructive lung diseases, and 28.4\% fibrotic lung disease. Mean FVC was 91.3\% predicted (SD 26.4\%), Mean FEV1 83.1\% (SD 28.9\%).

The model estimates were highly correlated with spirometric values for FVC $(\lambda=0.999)$, FEV1 $(\lambda=0.947)$, VC $(\lambda=$ 0.999), IC $(\lambda=0.997)$ and TV $(\lambda=0.962)$.

Univariate analysis demonstrated no patient characteristics predictive of discrepancy from spirometric values for FVC or VC.

Conclusions We describe a pilot data from the initial development of a new technique for non-invasively assessing lung volume and pulmonary function measurements. It correlates to within $30 \mathrm{ml}$ for FVC and $10 \mathrm{ml}$ for VC. This has a wide range of potential applications, including screening, home monitoring of respiratory disease, assessment of lung function in those unable to complete pneumotachygraphy and gating controls for radiological imaging techniques.
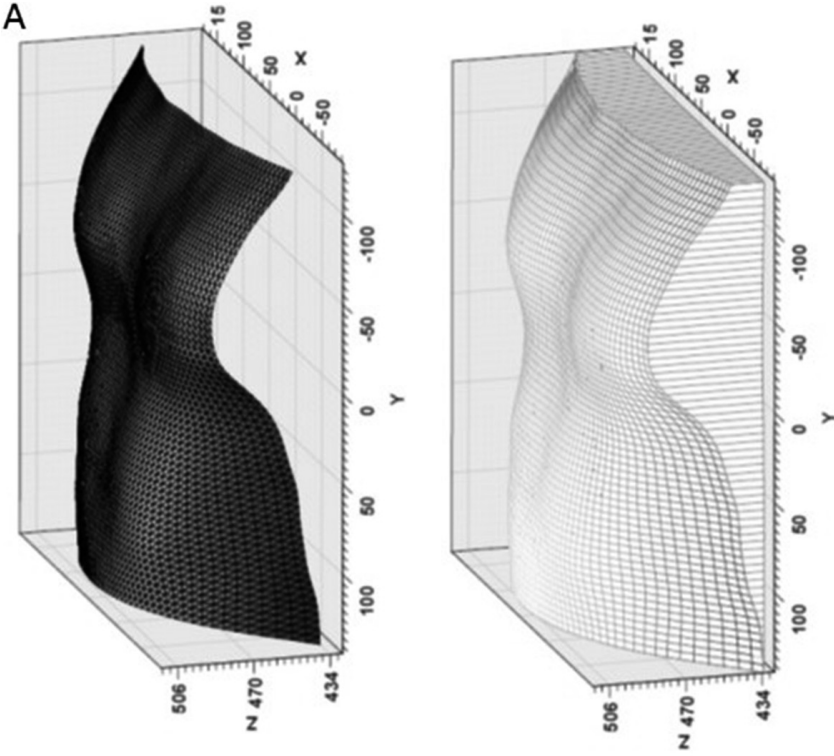

B
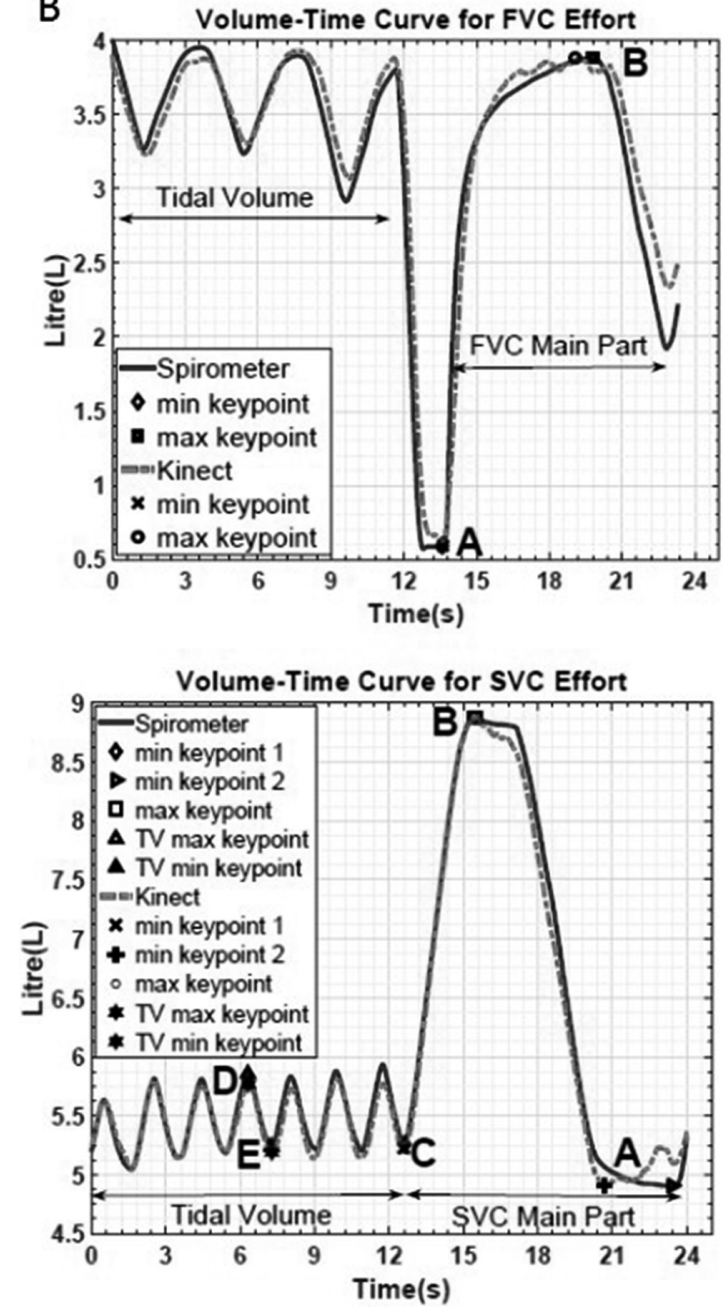

Abstract P83 Figure 1 (A) Reconstructed chest surface and volume estimation from reference plane. (B) Volume-time curves (FVC and SVC) comparing spirometer values and Kinect calculation

\section{REFERENCE}

1 Soleimani $\mathrm{V}$, Mirmehdi $\mathrm{M}$, Damen $\mathrm{D}$, et al. Remote pulmonary function testing using a depth sensor. Biocas 2015 